\title{
Starspots properties and stellar activity from planetary transits
}

\author{
Adriana Valio \\ Center for Radio Astronomy and Astrophysics (CRAAM) \\ Mackenzie Presbyterian University, Sao Paulo, Brazil \\ email: avalio@craam.mackenzie.br
}

\begin{abstract}
Magnetic activity of stars manifests itself in the form of dark spots on the stellar surface. This in turn will cause variations of a few percent in the star light curve as it rotates. When an orbiting planet eclipses its host a star, it may cross in front of one of these spots. In this case, a "bump" will be detected in the transit lightcurve. By fitting these spot signatures with a model, it is possible to determine the spots physical properties such as size, temperature, location, magnetic field, and lifetime. Moreover, the monitoring of the spots longitude provides estimates of the stellar rotation and differential rotation. For long time series of transits during multiple years, magnetic cycles can also be determined. This model has been applied successfully to CoRoT-2, CoRoT-4, CoRot-5, CoRoT-6, CoRoT-8, CoRoT-18, Kepler-17, and Kepler-63.
\end{abstract}

Keywords. Stars: activity, stars: rotation stars: spots

\section{Introduction}

Very likely, all cool stars with a convective envelope like the Sun will have spots on their surfaces. So far, a few thousand planets are confirmed to eclipse their host star, as observed by the CoRoT and the Kepler satellites. During one of these transits, the planet may pass in front of a spot group and cause a detectable signal in the light curve of the star.

To better study these stars spots, I developed a model that simulates planetary transits in front of stars (Silva 2003). In this model, the planet is used as a probe do determine the spots physical characteristics such as

- Size (area coverage)

- Intensity $\rightarrow$ temperature $\rightarrow$ magnetic field

- Location (longitude \& latitude)

- Lifetime

From the intensity of the spot with respect to the stellar center disc intensity, it is possible to estimate the spots temperature assuming that both the spot and the stellar photosphere irradiates as a black body. Once the temperature is known, the magnetic field of the spot can be inferred considering a solar like behavior and a temperaturemagnetic field relation such as the one given in Dicke (1970). According to this author the temperature varies with the magnetic field squared.

This model has already been successfully applied to stars CoRoT-2 (Silva-Valio et al. 2010, Silva-Valio \& Lanza 2011), Kepler-17 (Valio et al. 2017), and and Kepler-63 (Estrela \& Valio 2016). Other authors have also studied starspots properties. For example, the effect of the spots not occulted by the planetary transit was studied by Pont et al. (2013). Also, these same effects can influence the transmission spectroscopy measurements of the transit (Oshagh et al. 2014). Moreover, the detection of the same spot in consecutive transits can also provide information about the spin-orbit alignment of the planetary system (Sanchis \& Winn 2011, Sanchis et al. 2012). 
From spot detection on different transits, some stellar properties may also be determined, for example:

- Rotation period

- Differential rotation

- Activity cycle

The rotation period is calculated the same way as Thomas Harriot did for the Sun in 1610, that is, by tracing the sunspots "move" across the solar disc. This is done by detecting the same spot on a consecutive transit and noting how much its longitude has changed. Silva-Valio (2008) estimated the rotation period of HD 209458 applying this method.

Just as the Sun has an activity cycle of 11 years, so do other stars as shown by the studies of Baliunas et al. (1995), Saar \& Brandenburg (1999), Olah et al. (2009), Messina \& Guinan (2002). For long enough monitoring of stars with transits, it is possible to estimate the number of spots and its variation with the years. Since Kepler did observe thousands of stars for a period of 4 yeas, we may identify short duration cycles for some active stars (Estrela \& Valio 2016). For more details see article by Estrela \& Valio in this volume.

The next section presents the spot model used in this work, whereas the following section describes the studied stars. The results of the model such as spots physical parameters and the stellar rotational profile are described in Sections 4 and 5, respectively. Finally, the last section lists the conclusions.

\section{Planetary Transit Model}

The model used in this work simulates the passage of a planet (dark disc) in front of a star. For the star we can use an image of the Sun or a synthesised image of a star with limb darkening (see Figure 1):

$$
I=I_{0}\left[1-u_{1}(1-\mu)-u_{2}(1-\mu)^{2}\right]
$$

where $\mu=\cos (\theta)$, and $\theta$ is the heliocentric angle (or longitude if we consider the brightness variation along the equator). The parameters $u_{1}$ and $u_{2}$ define the decrease in brightness from the center to the limb. In the case of the Sun, $u_{1}=0.59$ and $u_{2}=0$, so the limb darkening is of linear form. For $u_{2} \neq 0$ then the stellar limb darkening is said to be quadratic.

The planet is an opaque circular disc of radius $r$ given in units of the stellar radius $R_{s}$ (black disc in the center of the star on Figure 1). In the case of a Jupiter-size planet orbiting a solar like star, $r / R_{s}=0.1$. The orbit of the planet is calculated using the period, $P_{\text {orb }}$, semi-major axis, a (also given in units of $R_{s}$ ), and the inclination angle, $i$. The orbit is considered circular (that is, zero eccentricity). If the orbital plane is parallel to the stellar equator, then the obliquity is null, otherwise, the obliquity parameter, $\lambda$, can be set in the model with the appropriate value. Once the orbit is set, then every 2 min (or the desired time interval), the planet is centred at its calculated position and all the pixels intensity in the image star + planet are summed, yielding the light curve. Thus the transit light curve of a spotless star is obtained, this is shown as the blue curve in the top panel of Figure 2.

An important feature of this planetary model is that it allows for the inclusion of spots on the stellar surface. These spots are described by three parameters:

- Intensity: measured with respect to stellar maximum intensity, $I_{0}$ (at the center);

- Size: measured in units of planetary radius $\left(R_{s}\right)$; 
Table 1. Stellar and Planetary Parameters

\begin{tabular}{lcccccccc}
\hline Star & CoRoT-2 & CoRoT-4 & CoRoT-5 & CoRoT-6 & CoRoT-8 & CoRoT-18 & Kepler-17 & Kepler-63 \\
\hline Spectral type & G7V & F8V & F9V & F9V & K1V & G9V & G2V & G8V \\
Mass $(M \odot)$ & 0.97 & 1.10 & 1.0 & 1.055 & 0.88 & 0.95 & 1.16 & 0.984 \\
Radius $(R \odot)$ & 0.902 & 1.17 & 1.19 & 1.025 & 0.77 & 1.0 & 1.05 & 0.901 \\
Prot (d) & 4.54 & 8.87 & 26.6 & 6.35 & 21.7 & 5.4 & 12.28 & 5.4 \\
Teff (K) & 5625 & 6190 & 6100 & 6090 & 5080 & 5440 & 5781 & 5576 \\
Age (Gyr) & $0.13-0.5$ & $0.7-2.0$ & $5.5-8.3$ & $1.0-3.3$ & $2.0-3$ & $?$ & $>1.78$ & 0.2 \\
\hline Planet & & & & & & & & \\
\hline Mass $\left(M_{\text {Jup }}\right)$ & 3.31 & 0.72 & 0.467 & 2.96 & 0.22 & 3.47 & 2.45 & - \\
Radius $\left(R_{\text {star }}\right)$ & 0.172 & 0.107 & 0.120 & 0.117 & 0.090 & 1.31 & 1.312 & 0.0662 \\
Porb (d) & 1.743 & 9.203 & 4.038 & 8.886 & 6.212 & 1.90 & 1.49 & 9.434 \\
a $\left(R_{\text {star }}\right)$ & 6.7 & 17.47 & 9.877 & 17.95 & 17.61 & 6.35 & 5.31 & 19.55 \\
\hline
\end{tabular}

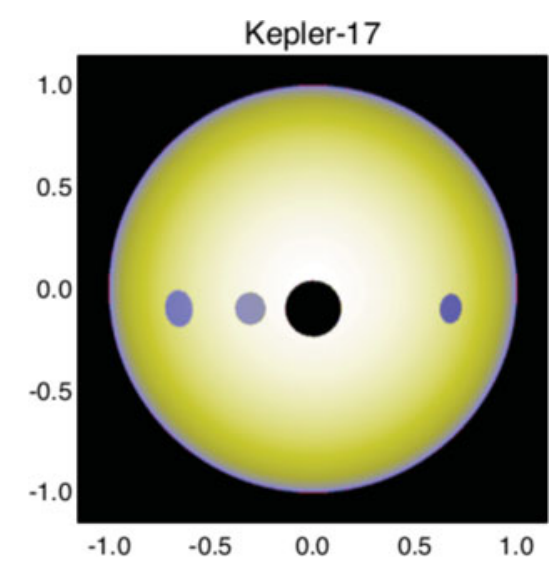

Figure 1. Planetary transit model.

- Position: Longitude and Latitude (restricted to the transit band).

The effect of foreshortenning as the spots approach the limb, a projection effect, is taken into account. An example of a star, Kepler-17, with three spots is shown in the top panel of Figure 1.

\section{Stellar observations}

Thus far we have analysed a set of 8 stars observed by the CoRoT and Kepler missiona, all of them solar like stars of spectral types F, G, and K, with varying ages. These stars have a Hot-Jupiter in close orbit around them with semi-major axis less than 20 stellar radii. The planetary mass vary from 0.22 to 3.5 Jupiter mass. The basic data of the stars and their planets are listed on Table 1.

\section{Spots characteristics}

The model described in Section 2 was applied to the 8 stars listed on Table 1. First the transits were cut from the continuous lightcurve, then we subtract a spotless star model from the light curve yielding the residuals. Figure 2 shows the transit lightcurve with the spotless model (blue curve) in the top panel, whereas the residuals after the subtraction is displayed in the bottom panel. The spots signature are clearly seen as peaks in the residuals. Each of these peaks identified in the transit light curve was modelled using a $\chi^{2}$-minimisation routine (AMOEBA). Only the peaks that exceed a certain threshold 

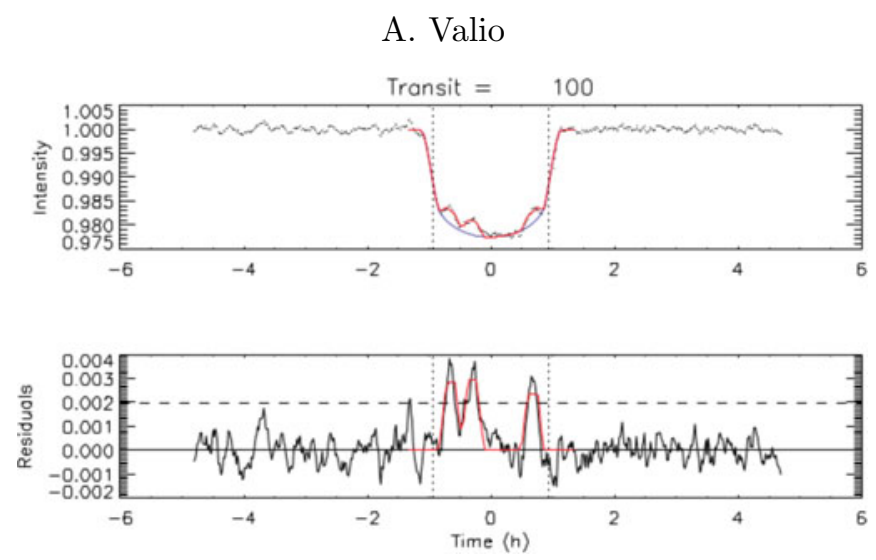

Figure 2. Top: Transit light curve with spotless star model overploted (red curve). Bottom: Residuals after spotless model subtraction from transit light curve, emphasising the spots signatures. The spot model is also shown.

were modelled. In the case of the CoRoT stars the threshold was 2 times the rms of the noise, whereas for the Kepler stars, this value equals 10 times the CDPP (Combined Differential Photometric Precision, Christiansen et al. 2012).

Each of the peaks, assumed to be caused by the passage in front of a spot, was modelled with the three parameters mentioned in Section 2, namely, size, intensity and position. The best fit of the model is shown as a red curve on Figure 2. The true size of the spot can be obtained once the size of the star, $R_{s}$ is known. The average size of spots (in millions of meters) are given in the first line of Table 2. For comparison the values for sunspots are also given in the last column.

Then the area covered by spots within the band of the stellar surface occulted by the planet during its transit can be measured. Usually, for the planets studied here the transit band has a latitudinal width of about $10^{\circ}$. The second line of Table 2 lists the average area covered by spots within the transit band.

The temperature of the spot may also be determined from the effective temperature of the star, $T_{e f f}$, assuming both the spot and the surface to emit as black bodies. For this purpose, the spot temperature, $T_{0}$, is calculated from the spot intensity, $f_{i}$, using:

$$
T_{0}=\frac{K_{b}}{h \nu \ln \left(f_{i}\left(e^{h \nu / K T_{e f f}}-1\right)+1\right)}
$$

where $K_{b}$ and $h$ are the Boltzmann and Planck constants, respectively, $T_{e f f}$ is the photospheric temperature given in the fourth line of Table 2 for each star, $\nu$ is the observation frequency corresponding to a wavelength of $600 \mathrm{~nm}$, and $f_{i}$ is the ratio of spot intensity with respect to the central stellar intensity $I_{c}$. The spot temperature calculated using Eq. 4.1 and averaged for all the spots of a given star is listed on the third line of Table 2 .

An interesting result from the spot temperature that we found is that the ratio of the spot temperature to the effective temperature of the stellar photosphere is basically constant and equal to 0.84 . This temperature ratio is presented in the last line of Table 2 , and also shown in the plot of Figure 3.

\section{Stellar rotation and differential rotation}

If the same spot is detected on a later transit, then the stellar rotation can be estimated by measuring its longitudinal displacement in time. Actually what is measured by the starspot movement is the rotation period at the transit latitude, $P_{\text {rot }}(\alpha)$, which may 
Table 2. Starspots Parameters

\begin{tabular}{|c|c|c|c|c|c|c|c|c|c|}
\hline Star & CoRot-2 & CoRoT-4 & CoRoT -5 & CoRoT-6 & CoRot-8 & CoRoT-18 & Kepler-17 & Kepler-63 & Sun \\
\hline Radius ( $\mathrm{Mm}$ ) & $55 \pm 19$ & $51 \pm 14$ & $75 \pm 17$ & $48 \pm 14$ & $82 \pm 21$ & $65 \pm 19$ & $80 \pm 50$ & $32 \pm 14$ & $12 \pm 10$ \\
\hline Area $(\%)$ & 13 & 6 & 13 & 9 & 29 & 13 & $6 \pm 4$ & $5 \pm 2$ & $<1$ \\
\hline Tspot $(\mathrm{K})$ & $4600 \pm 700$ & $5100 \pm 500$ & $5100 \pm 600$ & $4900 \pm 600$ & $4400 \pm 600$ & $4800 \pm 600$ & $5100 \pm 500$ & $4700 \pm 400$ & $4800 \pm 400$ \\
\hline Teff $(K)$ & 5625 & 6190 & 6100 & 6090 & 5080 & $54 \overline{40}$ & 5780 & 5580 & 5780 \\
\hline Tspot/Teff & 0.818 & 0.824 & 0.836 & 0.804 & 0.866 & 0.882 & 0.875 & 0.846 & 0.830 \\
\hline
\end{tabular}

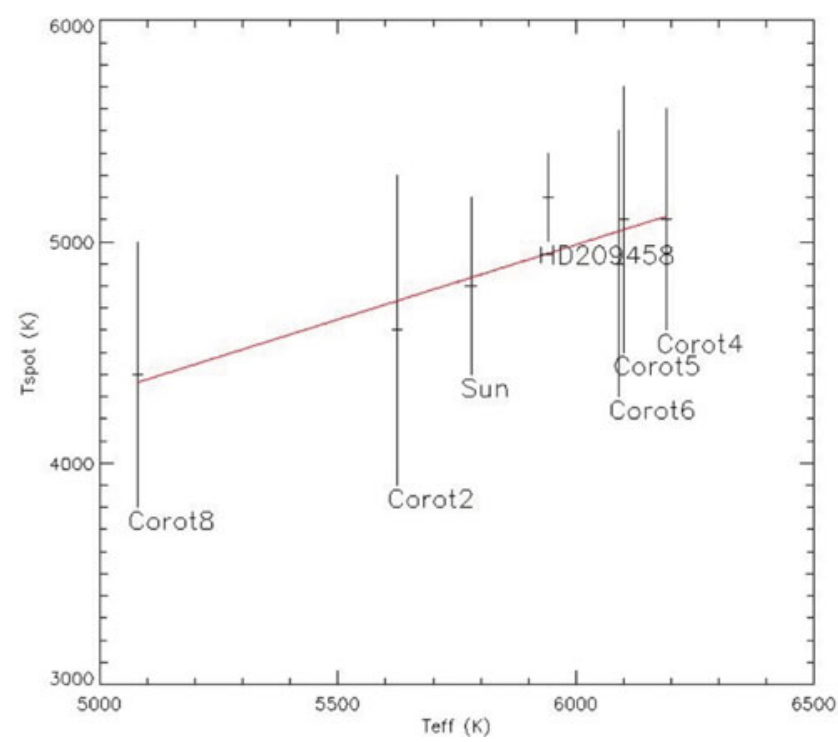

Figure 3. Spots temperature versus stellar effective temperature.

be different from the average rotation period of the star, $P_{\text {rot }}$ obtained from the whole light curve, neglecting the transits. To estimate this period, $P_{\text {rot }}(\alpha)$, we need the relation between the central time of the "bump" and the longitude on the surface of the star, as seen from Earth. The central time of the "bump", $t_{s}$ (in hours) is related to the longitude of the spot, $l g_{\text {spot }}$ by:

$$
l g_{\text {spot }}=\operatorname{asin}\left(\frac{\operatorname{acos}\left(90^{\circ}-360^{\circ}\right)\left(t_{s} / 24\right) / P_{\text {orb }}}{\cos \left(\text { lat }_{\text {spot }}\right)}\right)
$$

where $P_{\text {orb }}$ is the orbital period and $l a t_{\text {spot }}$ is the latitude of the spot in the stellar surface. The spot latitude is determined by the projection of the planetary transit onto the stellar disc, and is given by:

$$
\text { lat }_{\text {spot }}=-\arcsin [a \cos (i)]
$$

where $a$ is the planetary semi major axis and $i$ is the inclination angle of the orbit. The minus sign is arbitrarily chosen so that the planetary transit crosses the Southern hemisphere of the star.

Once the longitudes of the spots, $l g_{\text {topo }}$, as seen from Earth, on different transits is determined, we transform the longitudes of these spots to ones in the reference system that rotates with the star, $l g_{\text {ror }}$ :

$$
l g_{\text {rot }}=\lg _{\text {topo }}-360^{\circ} \frac{n P_{\text {orb }}}{P_{\text {rot }}(\alpha)}
$$


Table 3. Stellar rotation pattern

\begin{tabular}{lccccccccc}
\hline Star & CoRoT-2 & CoRoT-4 & CoRoT-5 & CoRoT-6 & CoRoT-8 & CoRoT-18 & Kepler-17 & Kepler-63 & Sun \\
\hline$P_{\text {rot }}(\mathrm{d})$ & 4.54 & 8.87 & 26.63 & 6.35 & 21.7 & 5.4 & 12.28 & 5.4 & 27.6 \\
Latitude, $\alpha\left(^{\circ}\right)$ & -14.6 & 0 & -47.2 & -16.4 & -29.4 & -22.8 & -4.6 & -48.5 & 0 \\
$P_{\text {rot }}(\alpha)(\mathrm{d})$ & 4.48 & 8.71 & 26.49 & 6.08 & 21.42 & 4.68 & 11.4 & - & 24.7 \\
$\Delta \Omega(\mathrm{rd} / \mathrm{d})$ & 0.042 & 0.026 & 0.103 & 0.101 & 0.014 & 0.45 & 0.077 & 0.133 & 0.050 \\
$\Delta \Omega / \bar{\Omega}(\%)$ & 3 & 3.6 & 43 & 10 & 5 & 38 & 15 & 11 & 22 \\
\hline
\end{tabular}

where $n$ is the transit number, $P_{\text {orb }}$ the orbital period, and $P_{\text {rot }}(\alpha)$ the rotation period of the star at the transit latitude. The main task here is to determine this rotation period, that is done by trying to find many spots on different transits with the same $l g_{r o t}$. For this purpose, we create a flux deficit function with respect to the spot longitude calculated for a certain $P_{\text {star }}$ according to Eq. 5.3. Then we calculate the auto correlation of this flux deficit function parametrised by $P_{\text {star }}$. The chosen value for the rotation period of the star at the transit latitude is that of the thinnest auto correlation function. This procedure is explained in detail in Valio (2013).

Table 3 list the stellar rotation period for the 8 stars studied and also for the Sun. The first line displays the average rotation period of the star that is usually obtained from a Lomb-Scargle periodogram, and is considered here to be an average period over all latitudes of the star. The latitude of planet center crossing, determined from Eq. 5.2 is given in the second line of Table 3 . The stellar rotation period at the latitude of the shadow of the planet as it transits, obtained as described above, is given in the third line of Table 3 .

The differential rotation of the star can be obtained by assuming a solar like (or antisolar like) rotation model of the type:

$$
\begin{aligned}
\Omega(\alpha) & =A-B \sin (\alpha) \\
A & =\Omega_{e q} \\
B & =\Delta \Omega=\Omega_{e q}-\Omega_{p o l e}
\end{aligned}
$$

where $\alpha$ is the stellar latitude, whereas the constants $A$ and $B$ are the rotation at the equator, $\Omega_{e q}$, and the rotational shear (or differential rotation), $\Delta \Omega$, respectively. To determine the two constants we use the two measured values of stellar rotation, $P_{\text {rot }}(\alpha)$ and $P_{\text {rot }}$, the averaged rotation values over all latitudes.

The rotational shear, $\Delta \Omega$, calculated from Eq. 5.6, is given in the fourth line of Table 3 , whereas the relative differential rotation, given by dividing the shear by the average rotation, $\bar{\Omega}$, is listed in the last line of Table 3 .

We note here that except for Kepler-63, all the planets are considered to orbit in a plane aligned with their host star equator, having null or very small obliquity as measured from the Rossiter-MacLaughin effect. This is not the case for Kepler-63 that is known to have an almost poleward orbit (Sanchis \& Winn 2011, Sanchis et al. 2012). For this star, the way to calculate the rotation differential rotation is more complicated and is explained in an article by Netto \& Valio in this volume.

\section{Conclusions}

Here we applied a planetary transit model that allows stellar spot modelling to 8 stars observed by the CoRoT and Kepler satellites (listed on Table 1). The model fits the peaks in the residuals of the transit light curves that are assumed to be the signatures of 
the spots when occulted by the planet. Three parameters are used to model each spot, namely, the size (in units of the stellar radius), intensity (with respect to the stellar central intensity) and position. From the intensity of the spots it is possible to infer their temperature using Eq. 4.1.

The results of the physical characteristics of the spots are displayed on Table 2. By comparing the average sizes of starspots with those of the Sun, we found these to be 4 to 7 times larger than sunspots. We believe that what we are measuring is actually active regions, or spot groups, and not individual spots, since the precision is not enough for that. After estimating the spots temperature from its intensity, an interesting result found was that the ratio between the mean spot temperature and the stellar photospheric temperature was basically constant and equal to $\sim 84 \%$. That is, hotter stars have, on average, hotter spots.

The rotation period of the stellar surface within the latitudes occulted by the planet was determined, and since this was different from the mean stellar rotation period estimated from the variations in the out-of-transit lightcurve (see Table 3), we concluded that the stars rotated differentialy. An estimate of this differential rotation may be done by considering a solar-like rotation profile, given by Eq. 5.5. The results yield stars with a shear comparable to the Sun, such as CoRoT-2 and Kepler-17, and others that show twice this shear such as CoRoT-5, CoRoT-6 and Kepler-63. As for the relative differential rotation, only CoRoT-5 and CoRoT-18 showed larger values than the solar ones.

With this work, we hope to have shown the many possibilities of the spot model presented here, developed in Silva (2003). That is, by modelling the small variations observed in the transit light curves one can determine:

- Spots physical characteristics: such as size, temperature, location, evolution or lifetime, surface area coverage, etc.

- Stellar rotation profile with differential rotation

- Magnetic activity cycles

From the known location of recurrent spots, one may infer the presence of active longitudes, a key ingredient to stellar dynamos. From the temperature of the spots it is also possible to estimate their average magnetic field, if we assume a relation similar to that of the Sun.

If the same spots are detected on multiple transits, then the rotation profile of the star can be constructed, as described in detail by Silva-Valio (2008), Silva-Valio et al. (2010), Silva-Valio \& Lanza (2011), and Valio (2017). For longer observing period, stellar activity cycles can be determined. Short magnetic cycles were estimated for two Kepler stars by Estrela \& Valio (2016) applying this method.

The results obtained from this model, such as spots characteristics, active longitudes, spots lifetime, and differential rotation, are crucial ingredients to the dynamo mechanisms believed to be at work on solar-like stars.

\section{References}

Baliunas, S. A., Donahue, R., Soon, W., Horne, J., Frazer, J., Woodard-Eklund, L., Bradford, M., Rao, L., Wilson, O., Zhang, Q., et al. 1995, ApJ, 438, 269

Christiansen, J. L., Jenkins, J. M., Caldwell, D. A., Burke, C. J., Tenenbaum, et al. 2012, PASP 124,1279

Dicke, R. H. 1970, ApJ, 159, 25

Estrela, R. \& Valio, A. 2016, ApJ, 831, 57

Messina, S. \& Guinan, E. F. 2002, A\&A, 393, 225

Olah, K., Kollath, Z., Granzer, T., Strassmeier, K. G., Lanza, A. F. et al. 2009, A 6 A, 501, 703 
A. Valio

Oshagh, M., Santos, N. C., Ehrenreich, D., Haghighipour, N., Figueira, P., Santerne, A., \& Montalto, M., 2014, A\& $A, 568$, A99.

Pont, F., Sing, D. K., Gibson, N. P., Aigrain, S., Henry, G., \& Husnoo, N. 2013, MNRAS, 432, 2917

Saar, S. H. \& Brandenburg, A. 1999, ApJ, 524, 295

Sanchis-Ojeda, R. \& Winn, J. N., 2011, ApJ, 743, 61

Sanchis-Ojeda, R., Fabrycky, D. C. , Winn, J. N., Barclay, T., Clarke, B. D., Ford, E. B. et al. 2012, Nature, 487, 449

Silva, A. V. R. 2003, ApJ (Letters), 585, L147

Silva-Valio, A. 2008, ApJ (Letters), 683, L179

Silva-Valio, A., Lanza, A. F., Alonso, R., \& Barge, P. 2010, A\&A A, 510, 25

Silva-Valio, A. \& Lanza, A. F. 2011, A $\mathscr{G} A$ 529, 36

Valio, A. 2013, Astronomical Society of the Pacific Conference Series, 472, 239

Valio, A., Estrela, R. Netto, Y., Bravo, J. P., \& de Medeiros, J. R. 2017, ApJ, 835, 294 